have described the association between air pollution and acute illness, however few studies have been conducted in Ireland to date. This study estimates the association between changes in mean daily air pollutant levels and daily acute hospital admissions in Dublin.

Methods Mean 24-hour levels of fine particulate matter $\left(\mathrm{PM}_{2.5}\right)$, coarse particulate matter $\left(\mathrm{PM}_{10}\right)$, and pollutant gases were calculated for the central Dublin area as a whole, using data from EPA monitors between 2007 and 2016. Daily total acute hospital admissions for respiratory and cardiovascular/cerebrovascular conditions were obtained from Healthcare Pricing Office records, for hospitals within the Dublin area, over the same period. Univariate analyses were performed, to investigate the association between individual pollutants and admissions, followed by analyses that mutually adjusted for several pollutants, with a lag of up to 2 days, to further describe the associations observed. Meteorological variables were controlled for in the model.

Results Univariate analysis revealed carbon monoxide as the strongest predictor of respiratory admissions. Multivariate analysis also identified sulphur dioxide as a strong predictor of respiratory admissions and $\mathrm{PM}_{2.5}$ and nitrogen dioxide as the strongest predictors of cardiovascular admissions.

Conclusion This study describes the extent of the impact of air pollution on hospital admissions and presents models for predicting future admissions with varying levels of pollution. The findings may inform policies to reduce urban air pollution, to develop early warning systems for the public and to improve the preparedness of hospital emergency departments on days of poor air quality.

\section{P82 CRITICAL DISCOURSE ANALYSIS OF THE JOINT COMMITTEE MEETINGS ON THE EIGHTH AMENDMENT OF THE CONSTITUTION OF IRELAND}

IS Shiplo, 1,2K O'Donoghue, 1,3S Meaney*. 'University College Cork, Pregnancy Loss Research Group, Cork, Ireland; ' ${ }^{2}$ niversity College Cork, The Irish Centre for Fetal and Neonatal Translational Research (INFANT), Cork, Ireland; ${ }^{3}$ University College Cork, National Perinatal Epidemiology Centre, Cork, Ireland

\subsection{6/jech-2019-SSMabstracts.233}

Background The 1983 Eighth Amendment to the Irish Constitution stated that the right to life of a pregnant mother and her unborn were equal, which meant that pregnancy terminations were illegal in Ireland. Ongoing debate surrounding strict pregnancy termination laws compelled the Irish government to form a committee tasked with deliberating the arguments for and against repealing the Eighth Amendment as well as forming recommendations. The objective of this study is to evaluate the issues raised and how information was presented during the joint committee meetings on the Eighth Amendment.

Methods Published transcripts from the Joint Committee on the Eighth Amendment of the Constitution meetings were accessed online for analysis. The committee's deliberations commenced on September 20, 2017 and concluded on December 14, 2017. This qualitative study applied a critical discourse analysis, which evaluates language in its social and cultural context. Critical discourse analysis allows for the deconstruction of language to gain an insight and understanding of socially produced meanings, recognising the ability for language to perpetuate inequalities and power dynamics.
Results Seven themes were identified from the meetings' contents related to pregnancy termination: including fatal fetal anomalies, rape and incest, decriminalisation, mental health, human rights, risk and practical issues. Throughout proceedings research evidence was often misrepresented and distorted in order to spin findings in support of members' arguments. Anecdotal evidence, including emotionally-laden narratives, was used to persuade members with divergent views to change their political and ideological opinions about what is socially desirable and acceptable. When discussing the risk and practical issues of introducing termination of pregnancy, the matter of conscientious objection and maternity hospitals whose governance are influenced by religious ethics were debated, clearly illustrating the influence of cultural and religious ideologies over the health of the population.

Conclusion The framing of the discourse throughout the meetings likely influenced the committee members' knowledge, perception, and understanding of key issues, consequently impacting the development of the pregnancy termination legislation introduced in 2019. This study demonstrates that healthcare is influenced by the political context and prevailing religious ideologies in Ireland. This study reveals the potential for underlying bias in political structures to sway healthcare legislation, which can impact health and in particular women's reproductive health.

\section{P84 IS SOCIAL ISOLATION AS BAD FOR HEALTH AS SMOKING 15 CIGARETTES PER DAY? FINDINGS FROM TWO LARGE PROSPECTIVE UK COHORTS}

RW Smith, I Barnes*, G Reeves, J Green, V Beral, S Floud. Nuffield Department of Population Health, University of Oxford, Oxford, UK

\subsection{6/jech-2019-SSMabstracts.234}

Background Social isolation has been associated with increased mortality, and a much-cited previous review stated that the risk is comparable to smoking 15 cigarettes per day. However, the available evidence is inconsistent. We examined social isolation in relation to all-cause mortality in two UK prospective cohorts, and assessed whether the excess risk associated with social isolation was comparable to that for smoking 15 cigarettes per day.

Methods After excluding people with vascular disease, cancer or low self-rated health, to minimise reverse causation bias, 326,169 Million Women Study (MWS) participants (mean age $=68$ years) and 296,913 UK Biobank (UKB) participants (mean age $=56$ years), were followed for death. Social isolation was measured using an index of self-reported frequency of contact with family or friends, social group contact, and living alone. Adjusted RRs for all-cause mortality were calculated using Cox regression, comparing most isolated participants to the least isolated. Analyses adjusted for smoking and 12 other possible confounding factors.

Results Within each cohort, about 12\% of participants were classified as most isolated and $44 \%$ as least isolated. Over 5.9 years of follow-up, 9667 MWS participants died; the most isolated had about a $30 \%$ excess risk of all-cause mortality compared to the least isolated $(R R=1.28,1.19-1.38)$. Over 6.8 years follow-up, 4694 UKB participants died; the most isolated had about a $40 \%$ excess risk of mortality compared to the least isolated $(R R=1.38,1.27-1.51)$. Of the constituent measures contributing to isolation, living alone was most 
consistently associated with an excess mortality. Previous analyses in MWS found smoking 15 cigarettes per day was associated with about a $180 \%$ excess risk of mortality compared to never smokers $(R R=2.77,2.72-2.82)$.

Conclusion While social isolation was associated with about a $30-40 \%$ excess risk of all-cause mortality, the excess risk associated with smoking 15 cigarettes per day was 4-6 fold greater, at around $180 \%$.

\section{P85 QUANTIFYING SOCIAL INEQUALITIES IN SERIOUS INFECTIONS IN ENGLAND: A LONGITUDINAL ECOLOGICAL ANALYSIS OF HOSPITAL EPISODE STATISTICS DATA}

1,2,3,4TE Wingfield, ${ }^{5} \mathrm{~T}$ Rose, ${ }^{5} \mathrm{~B}$ Barr, ${ }^{5} \mathrm{D}$ Taylor-Robinson*. ${ }^{1}$ Clinical Sciences and
International Public Health, Liverpool School of Tropical Medicine, Liverpool, UK; ${ }^{2}$ Institute
of Infection and Global Health, University of Liverpool, Liverpool, UK; ${ }^{3}$ Social Medicine,
Infectious Diseases, and Migration, Karolinska Institutet, Stockholm, Sweden; ${ }^{4}$ Tropical and
Infectious Diseases Unit, Royal Liverpool and Broadgreen University Hospitals NHS Trust,
Liverpool, UK; ${ }^{5}$ Institute of Population Health Sciences, University of Liverpool, Liverpool, UK

\subsection{6/jech-2019-SSMabstracts.235}

Background In England, infections cause $7 \%$ of deaths, cost 30 billion pounds annually, and are more common in disadvantaged populations. However, there has been no single, unifying analysis of the overall social patterning of the health burden of infections in England and how this is changing over time. This study quantified social inequalities in patients admitted to secondary healthcare facilities for infections in England between 2005 and 2016.

Methods An ecological analysis was performed using neighbourhood-level Hospital Episode Statistics (HES) data concerning emergency hospitalisations across England for the study period from 2005 to 2016. Linear mixed effects models were used to assess age-adjusted trends in emergency admission rates for serious infections. Absolute inequalities in emergency admission rates for serious infections between the most and least income deprived neighbourhoods were quantified and compared to trends in non-infectious disease related admissions.

Results Between 2005 and 2016, age-adjusted emergency admission rates for serious infections increased 52\% (1894 and 2870 per 100,000 population for 2005 and 2016, respectively) across England. In 2005, the absolute inequalities gap comparing rates of serious infections in the highest versus lowest deprivation quintile was 1197 per 100,000 (95\%CI 1176 to 1217 ) and this increased over time. The annual increase in absolute inequality in the most deprived areas compared to the least was 51 emergency admissions per 100,000 (95\%CI 49-52). In 2005, the absolute inequalities gap comparing rates of non-infectious disease related admissions in the highest versus lowest deprivation quintile was 5950 per 100,000 (95\%CI 5880 to 6020$)$. The annual increase in absolute inequality of non-infectious disease related admissions in the most deprived areas compared to the least was 27 emergency admissions per 100,000 (95\%CI 22-32). Subsequent analyses will explore trends in different age groups, alternative measures of inequalities, and sub-groups of infectious outcomes.

Discussion Emergency admission rates for serious infections and non-infections have increased in England. In addition, absolute inequalities have widened, especially for emergency admissions due to serious infections. These findings support the need for stronger public health and prevention efforts to address inequalities in the social determinants of ill health, with a specific focus on infectious diseases.

\section{P86 INTERPRETING THE CHANGING ASSOCIATION BETWEEN CAESAREAN BIRTH AND NEONATAL DEATH: A CASE STUDY FROM ETHIOPIA}

${ }^{1} \mathrm{E}$ Yisma*, ${ }^{2} \mathrm{~B} \mathrm{Mol},{ }^{1} \mathrm{~J}$ Lynch, 'L Smithers. 'School of Public Health, The University of Adelaide, Adelaide, Australia; '2Department of Obstetrics and Gynaecology, Monash University, Melbourne, Australia

\subsection{6/jech-2019-SSMabstracts.236}

Background Both individual- and aggregate-level studies have yielded inconsistent results about the association between caesarean birth and neonatal mortality. We provide an interpretation of the changing association over time between caesarean birth and neonatal death using Demographic and Health Survey (DHS) data within the context of Ethiopia.

Methods We used data from Ethiopian DHS in 2000, 2005, 2011, and 2016. We analysed the association between caesarean birth and neonatal death using log-Poisson regression models for each survey adjusted for potential confounders. We then applied the 'Three Delays Model' to provide an interpretation of the changing association between caesarean birth and neonatal death in Ethiopia.

Results The adjusted prevalence ratios (aPR) for neonatal death among neonates born via caesarean section versus vaginal birth increased over time, from 0.95 (95\% CI, 0.29, 3.19) in 2000 to $2 \cdot 81(95 \% \mathrm{CI}, 1 \cdot 11,7 \cdot 13)$ in 2016 . The association between caesarean birth and neonatal death was stronger among rural women (aPR (95\% CI) $3.43(1.22,9.67)$ ) and among women from the lowest quintile of household wealth (aPR (95\% CI) $7 \cdot 01(0 \cdot 92,53 \cdot 36))$ in 2016. However, the aggregate-level analysis revealed that an increase in caesarean section rate is correlated with a decrease in the proportion of neonatal deaths.

Conclusion The naïve interpretation of the changing association between caesarean birth and neonatal death from 2000 to 2016 is that caesarean section is increasingly associated with neonatal death. However, the changing association reflects improvements in health service coverage and a shift in the characteristics of Ethiopian women undergoing caesarean section after complicated labour or severe foetal compromise. 